\title{
Dual effects of spatial updating in both real and virtual environments
}

\author{
LIU ShuQing ${ }^{1}$, ZHENG XianJun Sam ${ }^{1 *}$, LIU XianYun ${ }^{2 *} \&$ PENG KaiPing ${ }^{1,3 *}$ \\ ${ }^{1}$ Department of Psychology, Tsinghua University, Beijing 100084, China; \\ ${ }^{2}$ Academy of Psychology and Behaviour, Tianjin Normal University, Tianjin 300074, China; \\ ${ }^{3}$ Department of Psychology, University of California, Berkeley, CA 94720, USA
}

Received June 2, 2012; accepted December 14, 2012

\begin{abstract}
When people move around in their environment, spatial updating, which is an automatic cognitive process, is essential to ensuring people can keep track of their relations between them and the surrounding objects, and to "recalculating" the relative position and orientation of those objects with regard to the current position of the persons. Despite the facilitating effect of spatial updating to people's mental representation in most circumstances as demonstrated in most of the existing literature, the effect sometimes can be adversarial. For instance, some research suggested that even though people were asked to ignore their locomotion, it is difficult to suppress updating of the spatial representation during movement. The current two studies were conducted to systematically investigate the dual effects of spatial updating in both real and virtual environments. We used a typical spatial updating paradigm to explore the effects of scene familiarity (familiar vs. novel) and person's locomotion (stationary vs. moving) on change detection accuracy (target object moved or not). The results indicated a facilitating effect of spatial updating in the novel scene condition, but an adversarial effect in the familiar scene condition — the dual effects, in both real and virtual environments.
\end{abstract}

spatial updating, spatial representation, scene recognition, virtual environment, virtual reality

Citation: $\quad$ Liu S Q, Zheng X J S, Liu X Y, et al. Dual effects of spatial updating in both real and virtual environments. Chin Sci Bull, 2013, 58: 898-906, doi: $10.1007 / \mathrm{s} 11434-013-5676-7$

When people move around in their environment, the change of their position and orientation will lead to a change of spatial relations between themselves and the objects in the environment. In order to recognize and act on the objects from the new position or orientation, people need to keep track of their relationship with the surrounding objects. This cognitive process is known as spatial updating [1-4]. For example, when a person turns right, the cap originally placed in front of him or her now appears to his/her left. In order to remember and reach the cap later, he or she needs to keep track of the position of the cap when he or she moves. Spatial updating enables the person to calculate the location and orientation of the cap during his/her movements.

*Corresponding authors (email: sam.zheng@gmail.com; liuxianyun5099@163.com; kppeng@berkeley.edu)
Indeed, spatial updating plays an important role for us to experience a stable visual world during movements. If all the objects around us can be seen directly, this process may not be so important. However, once some objects are out of sight, the process of spatial updating becomes critical to ensuring that people can remember the position and orientation of those objects.

In the field of spatial cognition, it is important to study the spatial updating of scene recognition across views for the following reasons. First, we rarely perceive objects by themselves alone; instead we perceive objects together with their surrounding contexts. Hence, the online spatial updating is required to represent the environment we live in [5]. Second, people appear to be more sensitive to perceive changes to the layout than changes to individual object or object features [6]. Third, people's movements and spatial updating often are associated with the representation and recognition 
of the configuration or layout of objects in daily life [7].

Previous studies have shown that spatial updating in longterm memory is automatic and effortless, often referred as "automatic spatial updating" [8-10]. The spatial updating occurs automatically during our physical motion without requiring attention. For example, when people, who were blindfolded or in darkness, were asked to point to the previously well-learned targets relative to their new position after moving, their response latencies and/or accuracy rates were either not affected or only slightly affected by their prior locomotion [2,11-13].

Moreover, some research studies have indicated that spatial updating is reflex-like, hard-to-suppress, and largely beyond the conscious control of cognitive processes. It is difficult to ignore the updating of our egocentric spatial representation during movement. This phenomenon is called obligatory spatial updating, a subset of automatic updating [14]. For instance, compared with the updating condition, response latencies increased and/or accuracy rates declined when participants were required to ignore the previous locomotion and imagine they were still standing in the initial position, and then to point or walk to the target. Their performance was better in the updating condition than in the ignore condition. This indicated that spatial updating is hard to suppress when people move to new locations and orientations $[8,9,15]$. The results support the notion that updating can occur involuntarily and that it is hard to ignore.

In addition, studies have shown that locomotion plays an important role in automatic spatial updating. In everyday life we can perform spatial updating by using external cues such as visual surroundings and optic flow, or internal cues such as changes in vestibular and proprioceptive information during physical movement. Some research provided evidence that the response latencies and accuracy rates were quite similar only using vestibular and proprioceptive cues when participants were blindfolded in the updating condition. On the contrary, spatial updating could be severely interfered with if vestibular and proprioceptive cues were impaired $[13,15,16]$. Thus, vestibular and proprioceptive information cues were considered necessary and sufficient for automatic spatial updating and/or obligatory spatial updating.

In addition to the aforementioned studies which showed that the spatial updating of a well-learned scene is automatic and hard-to-suppress, several studies also suggested that the spatial updating of a briefly viewed scene can also be automatic.

Simons and Wang $[17,18]$ reported that scene recognition of a briefly learned layout was view independent or unaffected after people walked around the layout. They used the typical spatial updating paradigm to compare both the influence of observers' locomotion and the rotation of layouts on the performance of scene recognition. They had participants briefly view an array of five objects on a desktop for $3 \mathrm{~s}$ in a dark environment and then detect the position change of one object after $7 \mathrm{~s}$. Participants were tested either from the learning perspective or from a new perspective. The results showed that visual detection of an object's position change at a novel view was less impaired when the novel view was caused by the observer's locomotion than when the novel view was caused by the table rotation. And this phenomenon could be observed even when participants used a handle fixed on the table to actively control the layout rotation. They argued that observers update the scene representation automatically during their movement. This updating mechanism which is independent of the visual information can be available only through vestibular information and proprioceptive information, and the process barely occupies cognitive resources and generates errors.

However, the studies of Simons and Wang [17,18] did not entirely rule out the possibility that object locations may also be represented according to the visual cues in the environment. Although participants were blindfolded or closed their eyes in the dark, this method cannot ensure that the visual cues or visual adaptation were completely ruled out. For instance, participants may obtain visual information occasionally or use background information because of their dark adaptation. Some evidence has proven that recognition accuracy can be increased when provided with an external or internal landmark or a cue in the dark environment [19-22]. In particular, Burgess et al. [23] used the typical spatial updating paradigm to examine whether the location representation of objects is affected by the environmental cues outside of the layout. They found that the recognition performance was better when the scene and the external cue rotated together compared to the condition when the scene rotated individually. Thus, people can represent the objects relative to external landmarks and this kind of cue may contribute to the scene change detection over brief learning and short distance. Moreover, Simons and Wang's studies $[17,18]$ have not indicated whether spatial updating could be ignored or not. The design of their experiment was not targeted at exploring systematically the dual effects of spatial updating.

Even though most of the above mentioned studies were conducted in real environments, spatial updating in virtual environments is also interesting for a number of reasons. First, the advanced virtual reality (VR) technology can generate high-fidelity visual environments that allow researchers to replicate and validate their experiments which were conducted in real environments. Second, VR can generate a variety of novel environments or stimuli (e.g. a fire scene) that are difficult or too risky to produce in the real environment. Lastly, VR can be used to design experiments with full control of the environment, eliminating the influence of other irrelevant factors [24-27]. For instance, in the real environment, visual clues and non-visual cues provided by our locomotion are difficult to separate. However, virtual reality technology provides a suitable method for exploring the effects of one or several combined factors separately.

By using of the circular screen combined with the im- 
mersive virtual environment technology, Lehmann, et al. [28] explored the influence of spatial updating on scene recognition tasks in an environment with strong and continuous cues. They used a screen to show the external cues and used the same experiment procedure as of Simons and Wang [17]. In the testing phase, participants pointed out the object where its position was changed on the table. Every participant completed four different testing conditions; they were two viewing position (unchanged or changed) combined with the retinal projection (same or different). The rotation magnitude of table and passive locomotion magnitude were both $50^{\circ}$. The results showed a view dependency effect when participants were at the unchanged position, which meant the change detection performance was impaired if the layout was rotated. On the contrary, the change detection performance at a changed view was not significantly impaired when the novel view was caused by the locomotion of the observer, so the moving of participants reduced the cost of mental rotations. This result replicated the findings of Simons and Wang [17]. However, their study did not remove external cues completely to explore the hypothesis that the spatial updating was only caused by the locomotion, and it did not investigate the dual effect of spatial updating, especially the interference effect in the virtual reality environment.

The main goals of our study are to examine the following questions: (1) whether spatial updating has dual effects, especially the interference effect on spatial reasoning after briefly learning a previous-viewed scene (Experiment 1); (2) whether the dual effect of spatial updating also occurs in the virtual reality environment when the external environmental cues were excluded (Experiment 2); and (3) whether VR technology has good validity in researching spatial updating of scene recognition. We applied the typical spatial updating paradigm as in Simons and Wang's study [17], but with a refined experimental design in order to address our research questions above. We hypothesized that locomotion-induced spatial updating facilitates scene recognition if participants perform better at the novel scene condition, but an interference effect of locomotion at the familiar scene condition (Experiment 1). We also hypothesized the similar result patterns will occurred in the virtual environment (Experiment 2).

\section{Experiment 1}

The main purpose of this experiment was to examine whether spatial updating has dual effects, especially the interference effect on spatial reasoning after a brief learning of a previously-viewed scene.

\subsection{Method}

(i) Participants. Thirty-two university students at a major Chinese university (16 males and 16 females, with normal visual acuity) were recruited to participate in the study and received monetary compensation.

(ii) Materials. The experiment was carried out in a room (6 m long, $6 \mathrm{~m}$ wide, $2.5 \mathrm{~m}$ high) with all walls covered with black curtains. A circular table covered by a grey mat (69 $\mathrm{cm}$ above the floor, $80 \mathrm{~cm}$ in diameter) and two chairs (seated $42 \mathrm{~cm}$ high) were placed in the center of the room, with 5 objects (around $5 \mathrm{~cm}$ in size) placed on the table. The five objects were randomly placed in five of nine locations and formed an irregular layout on the table. The distance between any two adjacent locations were from 19 to $30 \mathrm{~cm}$. The distance from the chairs to the center of the table was $90 \mathrm{~cm}$. The angle between the two chairs was $49^{\circ}$. Participants were asked to wear a blindfold and a wireless earphone. The lights were kept off during the whole experiment, and the experimenter only used a flashlight when arraying the objects. Throughout the experiment, participants could only see the positions of the five objects. We used the earphone to present white noise and instructions.

The experimenter created 40 irregular layouts of object locations. In each location, one of the five already occupied positions was randomly selected to be the position of the moved target object. The target object was moved to one of the remaining four positions. The new position of the target object was usually closest to the original position and had the same distance to the middle of the table so that the cues of distance were excluded.

(iii) Design. The independent variables were scene familiarity (familiar scene vs. novel scene) and person's locomotion (person stationary vs. person moving). Person's locomotion was manipulated within participants and scene familiarity was manipulated between participants. The locomotion angle of participants and the rotation angle of the table were both $49^{\circ}$ (Figure 1). The dependent variable was the percentage of the correct judgments of the locomotion position of the target object.

(iv) Procedure. With the assist of the experimenter, the blindfolded participants entered the room and sat in the viewing chair. Each trial consisted of three stages: Practicing, learning, and testing, as shown in Figure 2.

In the practicing phase, blindfolded participants practiced for about 15 min until they could stand up or sit down from a chair and walk to the other chair and had 10 extra trials ( 5 for the person moving condition) as practice to be familiar with the procedure. In the learning phrase, the experimenter pressed a key to start the experimental program, and then participants heard the instruction through an earphone. Participants should remove the blindfold and learn a layout of five objects. After $3 \mathrm{~s}$, participants were instructed to wear the blindfold and walk to the new position or keep still at the learning location. After $10 \mathrm{~s}$ participants learned the layout, they should remove the blindfold and judge which object was moved as accurately as possible. After the judgment, the experimenter pressed a key to end the trial and the verbal instruction guide participant to wear the blindfold and 

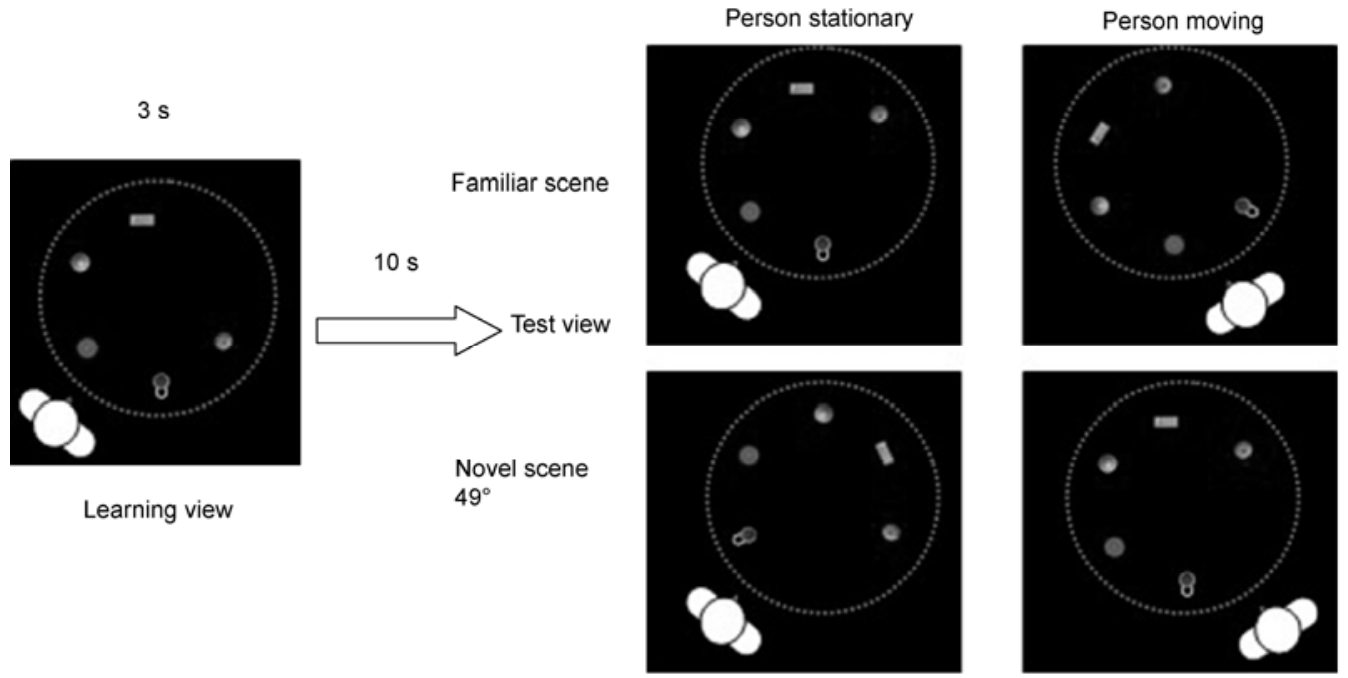

Figure 1 Experiment design in real environment.
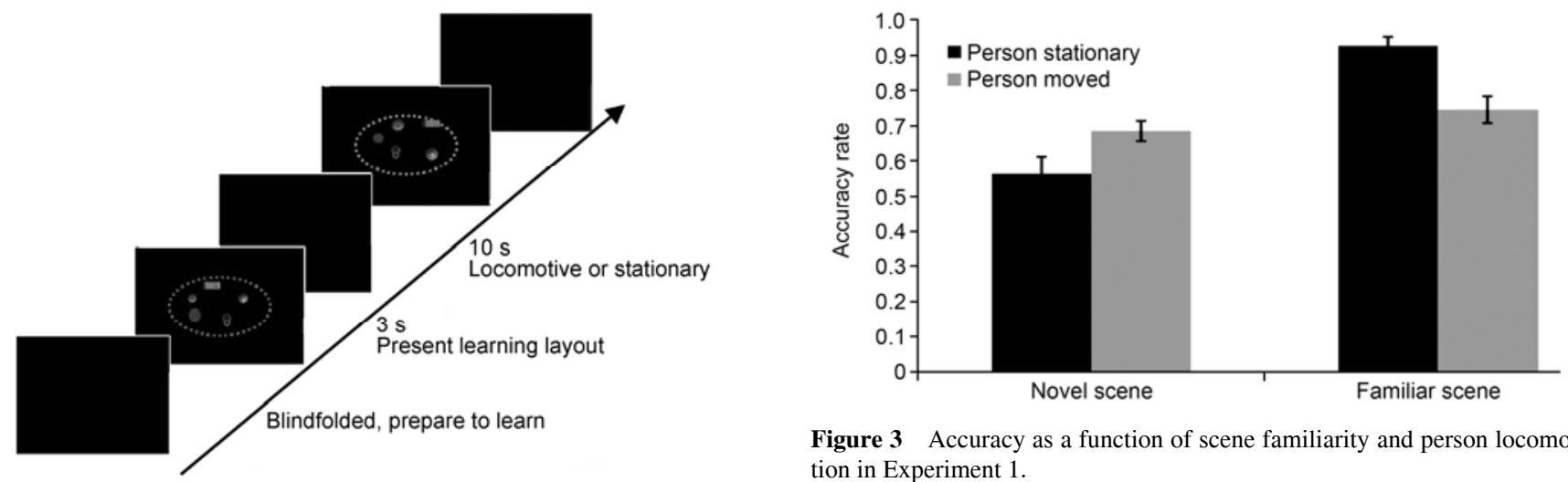

Figure 3 Accuracy as a function of scene familiarity and person locomotion in Experiment 1.

Figure 2 Sequence of presenting stimuli in a trial in real environment.

go back to the learning position and sit on the chair for the next trial. Each participant completed 40 trials.

\subsection{Results}

Accuracy rate of judgments as a function of scene familiarity and person's locomotion is shown in Figure 3. We analyzed data by use of mixed analyses of variance (ANOVAS); person locomotion was within participants and scene familiarity was between participants.

The main effect of scene familiarity was significant, $F$ (1, $30)=20.56, P<0.01$. The main effect of person locomotion was not significant, $F(1,30)=2.94, P>0.05$. The interaction between person locomotion and scene familiarity was significant, $F(1,30)=38.54, P<0.01$. Participants facing the familiar scene condition were more accurate than participants facing the novel scene condition, and there were no accuracy differences between participants in the person stationary condition and in the person locomotion condition. However, participants facing the novel scene were more accurate in the person locomotion condition than in the person stationary condition, and participants facing the familiar scene were also more accurate in the person stationary condition than in the person locomotion condition.

The primary purpose of Experiment 1 was to exam whether spatial updating has a dual effect, especially the interference effect on spatial reasoning after briefly learning a previousviewed scene. The result indicated the facilitating effect of spatial updating occurred because participants' performance was better in the person locomotion condition than in the person stationary condition in the novel test view. At the same time, the interference of spatial updating also appeared since participants in the familiar test view were more accurate in the person stationary condition than those in the person locomotion condition. So the results are consistent with our hypotheses about the dual effect of spatial updating in a real environment.

\section{Experiment 2}

The main purpose of this experiment was to investigate whether the dual effect of spatial updating occurs in the virtual 
reality environment without the external environmental cues.

\subsection{Method}

(i) Participants. Thirty-two university students at a major Chinese university (16 males and 16 females, with normal visual acuity) were recruited to participate in the study and received monetary compensation.

(ii) Materials. The virtual reality system (Figure 4) include a head-mounted display (HMD), used in conjunction with a rendering computer and a head tracking system. The head tracker determines the position and orientation of the user and the information is sent to the computer. The rendering computer generates and outputs three-dimensional visual and auditory imagery back to the user's HMD according to the position and orientation of the user's head. The HMD consists of earphone and video displays. This kind of virtual reality system is the most widely-used virtual technology in psychology study [24,26]. In our study all the three-dimensional virtual scenes were created by use of the 3-D MAX software and presented through the portable head- mounted display (HMD, I-O Display Systems, Inc. California). The IS-900 moving tracking system (InterSense, Inc., Massachusetts) could determine participants' head position in real environment. Virtual reality input devices were placed in a room (6 m long, $6 \mathrm{~m}$ wide, $2.5 \mathrm{~m}$ high). The HMD presented identical images to both eyes. The resolution is $800 \times 600$ pixels and the field of view (FOV) is $31^{\circ}$ diagonally for each eye. The virtual scenes were programmed with an ATI Radeon $\times 300$ graphics accelerator; updating the graphics and display at $100 \mathrm{~Hz}$. Whenever participants looked at the center of the unseen table through the HMD, participants could see the virtual scenes (or a red arrow before the scene was presented) from the centre of the FOV. The distance from the learning position or the testing position to the center of the unseen table was $1.9 \mathrm{~m}$.

All the walls of the experiment room were covered with black curtains. In the virtual environment participants saw the layouts which were composed of 5 virtual objects on the unseen table $(80 \mathrm{~cm}$ in diameter, $20 \mathrm{~cm}$ above the floor). The objects were generated by the 3-D MAX software and placed on five of nine locations in an irregular array similar to Experiment 1. The distance between any two adjacent locations were from 19 to $30 \mathrm{~cm}$. The experiments were conducted in a dark environment, so participants could see

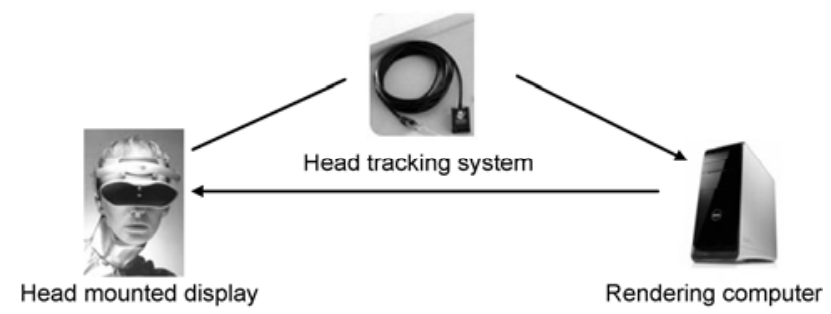

Figure 4 The virtual reality system. only five objects and their locations.

Just as in Experiment 1, the program created 40 irregular layouts of object locations. In each location, one of the five already occupied positions was randomly selected to be the position of the moved target object. The target object was moved to one of the remaining four positions. The new position of the target object was usually closest to the original position and had the same distance to the middle of the unseen table so that the cues of distance were excluded.

(iii) Design. The independent variables were scene familiarity (familiar scene vs. novel scene) and person's locomotion (person stationary vs. person moving). Person's locomotion was manipulated within participants and scene familiarity was manipulated between participants. The locomotion angle of participants and the rotation angle of the scene were both $49^{\circ}$ (Figure 5). The dependent variable was the percentage of the correct judgments of the locomotion position of the target object.

(iv) Procedure. With the assist of the experimenter, the blindfolded participants entered the room and stood in the learning position facing the virtual table scene. Each trial consisted of three stages: Practicing, learning and testing, as shown in Figure 6 . In the practicing phase, participants wearing the blindfold and the helmet walked along the marked radian assisted by the experimenter and practiced for about $15 \mathrm{~min}$ until they could walk properly along the marked radian. Then the experimenter helped participants remove the blindfold, and went behind the black curtains, opened the tracker and started the program. Participants had 10 extra trials ( 5 for person moving condition) as practice to be familiar with the procedure.

In the learning and testing phrase, the participant first stood in the learning position and looked at the scene through the HMD. In the center of the visual field, a red vertical arrow was presented to indicate the position of the forthcoming layout. The experimenter presses a key to start each trial, and then participants were told to pay attention to the position of the red arrow vocally through the earphone of the HMD.

Then the red arrow disappeared and the instruction reminded the participants through the earphone that they should try to remember the locations of the objects they were going to see. Then participants learned a layout. After $3 \mathrm{~s}$, the layout disappeared. Then participants were instructed to walk to the new position or keep still at the learning location. After $10 \mathrm{~s}$ a test scene was presented and participants were instructed to judge which object was moved as accurately as possible. After the judgment, the experimenter pressed a key to end the trial and the verbal instruction guided participants to go back to the learning position for the next trial. Each participant completed 40 trials.

\subsection{Results}

As in Experiment 1, accuracy rate of judgments was a 
$3 \mathrm{~s}$

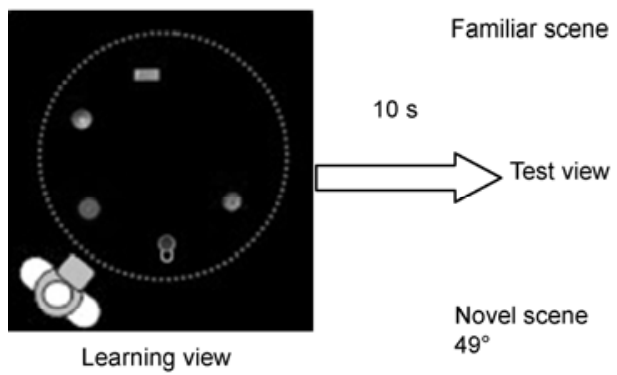

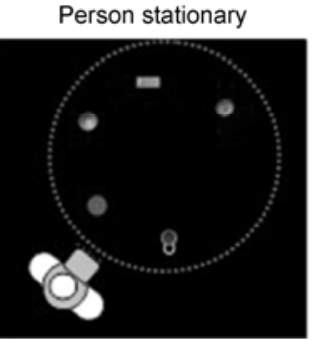

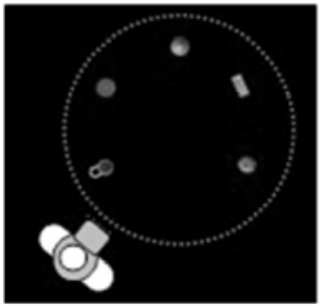

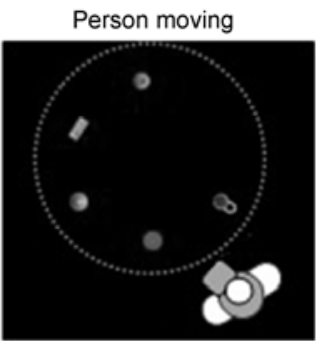

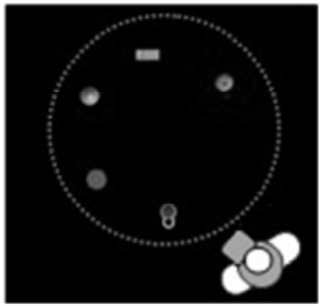

Figure 5 Experiment design in virtual environment.

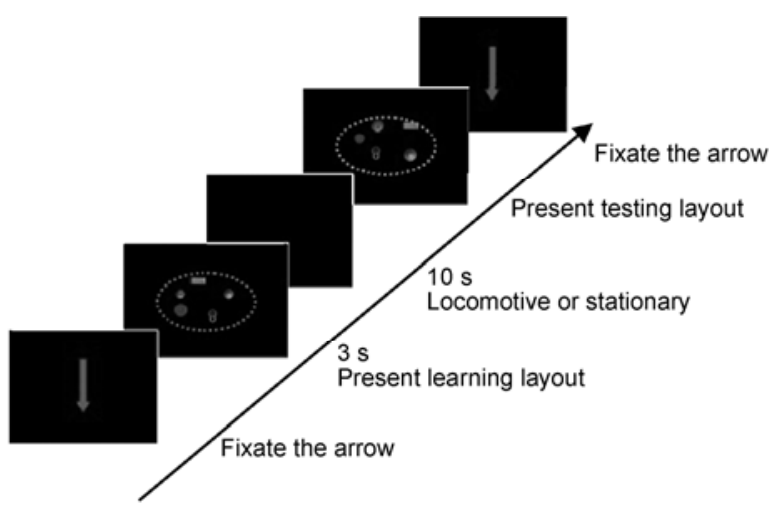

Figure 6 Sequence of presenting stimuli in a trial in virtual environment.

function of scene familiarity and person's locomotion (Figure 7). We analyzed data by use of mixed analyses of variance (ANOVAS); person locomotion was within participants and scene familiarity was between participants.

The main effect of scene familiarity was significant, $F(1$, $30)=23.21, P<0.01$. The main effect of person locomotion was not significant, $F(1,30)=1.21, P>0.05$. The interaction between person locomotion and scene familiarity was significant, $F(1,1)=17.94, P<0.01$. Participants facing the familiar scene condition were more accurate than participants facing the novel scene condition, and there were no accuracy differences between participants in the person stationary condition and in the person locomotion condition. However, participants facing the novel scene were more accurate in the person locomotion condition than in the person stationary condition, and participants facing familiar scene were also more accurate in the person stationary condition than in the person locomotion condition.

The primary purpose of Experiment 2 was to explore whether the dual effect of spatial updating occurs in the virtual reality environment without the external environmental

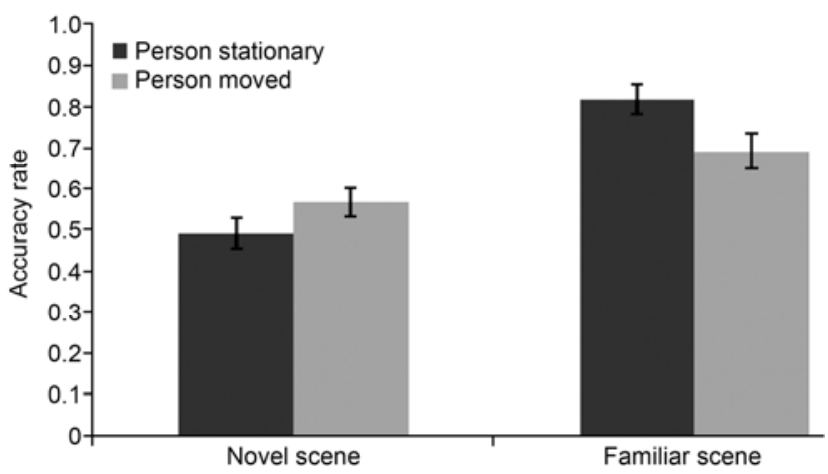

Figure 7 Accuracy as a function of scene familiarity and person locomotion in Experiment 2.

cues after briefly learning a previously-viewed scene. The result also indicated that the facilitative effect of spatial updating occurred because participants in the novel test view were more accurate in the person locomotion condition than in the person stationary condition. At the same time, the interference of spatial updating also appeared since participants in the familiar test view were more accurate in the person stationary condition than those in the person locomotion condition. So the results are consistent with our assumptions about the dual effect of spatial updating in a virtual reality environment.

In addition, we used an independent sample $T$-test to investigate the relationship between the performances in the real environment and in the virtual environment. There was a significant difference in the result between the two environments, $T(62)=1.99, P=0.05$, that is to say, participants in the real environment were more accurate than those in the virtual environment on the whole. Nevertheless, accuracy rates have the same reaction model in two environments, because the correlation coefficient of accuracy rates pattern between real and virtual environments was significant, $R=$ 
$0.98, P<0.01$. The result accorded with our expectations.

\section{Discussion}

The primary goals of these two studies were to investigate (1) whether spatial updating has dual effects, especially the interference effect on spatial reasoning after briefly learning a previously-viewed scene (Experiment 1), (2) whether the dual effect also occurs in the virtual environment when the external environmental cues were completely excluded (Experiment 2), and (3) whether VR technology we employed in the study has good validity in conducting research on spatial updating of scene recognition (Experiments 1 \& 2).

We used the classic spatial updating experimental paradigm as Simons and Wang's study [17], but improved the experimental design to systematically examine the dual effects of spatial updating. The results showed in the novel test view condition, participants' performance was better in the locomotion condition than in the stationary condition in both real and virtual environments-a facilitating effect of spatial updating as shown by many spatial updating studies. However, during the familiar test view condition, participants' performance was worse in the locomotion condition than in the stationary one also in both real and virtual environments-an adversarial effect of spatial updating. Even though, the performance in the real environment was more accurate as compared to the performance in the virtual environment, the results patterns in both environments are consistent. These results supported our initial hypothesis regarding the dual effects of spatial updating, and also indicated that the VR technology used in our study has good validity.

Although past research has demonstrated that spatial updating in long-term memory is automatic and effortless [8-10,13,15], our studies results contribute to the literature with the following aspects. First, we provided additional evidence that spatial updating for a briefly previous-viewed scene is also automatic, and cannot be ignored. Second, we improved the experimental design and the classic paradigm of spatial updating in scene recognition. Third, we confirmed the dual effects of spatial updating also happens in virtual environments, even though the previous VR study by Lehmann et al. [28] failed to address this question.

The dual effects of spatial updating can be explained by the allocentric updating model [29-31]. According to this model, after studying a layout in a brief time, participants were likely to represent the spatial relationship between objects and their' own position and orientation with respect to the intrinsic reference frame of the scene layout. Although the participants in the novel test view received the same retinal projection of scenes, their recognition accuracy in the locomotion condition was much higher than those in the stationary condition. This indicated spatial updating facilitated the scene recognition. In the stationary condition, participants adjusted the relationship between their own bodies and the intrinsic reference frame of the scene by use of mental rotation, and this process requires computation and inference; whereas the participants in the locomotion condition automatically updated the spatial relationship between their bodies and the intrinsic reference frame according to the associated vestibular and proprioceptive information provided via the movement. This may account for the facilitating effect of spatial updating. Likewise, though participants in the familiar test view also received the same retinal projection of scenes, their recognition accuracy in the locomotion condition was much worse than in the stationary condition, indicating that spatial updating during locomotion interfered with the accuracy of recognition. Most likely, participants automatically updated their position and orientation with respect to the intrinsic reference frame of the scene layout during their locomotion, and this process could not be ignored. So the expected scene representation was inconsistent with the scene they actually saw, which led to the decrease of recognition accuracy rates.

In addition to the dual effects of spatial updating, both experiments' results also showed that the viewpoint dependency effect on the scene recognition task in both real and virtual environments. No mater participants walked to the new viewing position or remained stationary at the learning position, their performance in the familiar scene test view was more accurate than that in the novel scene test view. Therefore, spatial updating during locomotion does not eliminate viewpoint-dependent visual scene processing. These findings are inconsistent with the findings of Simons and Wang [17], where they found that recognition performance at a novel view was much impaired when the novel view was being caused by the passive table rotation, and yet detection performance at a novel view was hardly impaired when the novel view was caused by the locomotion of the observer. We think this inconsistent findings may caused by different experiment designs used between their studies and ours. In order to test the view dependent effect, we should compare the condition in which participants and table were relatively still and the condition in which the participant remained stationary while the table was rotated, or vice versa. Also, as to examine whether the dual effect occurs, we ought to compare the performances in person locomotion condition with person stationary condition for the novel test view, and also compare the performances in person locomotion condition with person stationary condition for the familiar test view. The refined experimental design allowed us to clearly examine the view dependent effect and also the dual effects of spatial updating.

In our study, there was a very significant correlation of the behavior performance between the two environments. The consistent result patterns in both real and virtual environments also indicate that the spatial updating ability could be transferred to a new situation in a short time. In addition, 
the correlation coefficient between the two environments is comparable with other studies [32,33], and even better than some studies $[34,35]$. This demonstrates that VR technology has good validity in the research on spatial updating of scene recognition. However, the performance in the real environment was higher than those in the virtual environment on the whole. The possible reasons are as follows. First, we were unable to rule out completely the visual cues or visual adaptation that participants could obtain in the real environment. Second, although the VR technology we used creates high fidelity 3-D graphics, it also suffered from technology limitations, such as imperfections in the rendering models, limitations of the visual display (low spatial resolution, small field of view), slow graphics update rate, and lags between head tracking and visual display, etc. All these factors could potentially contribute to the overall declines of accuracy rates in the virtual environments.

Despite the limitations of VR technology, our study results showed its good validity. For future research, we will consider to use VR to investigate the following questions. First, whether the dual effects of spatial updating on the visual scene can be affected by the angular distance of the locomotion. Also can we replicate spatial updating research in much large environment, such as the room size layout, instead of a desk size layout? Or can we explore the physiological mechanism on the dual effects of spatial updating using VR technology?

In summary, the present two studies demonstrate that spatial updating during locomotion facilitates visual scene recognition in novel views, but interferes with visual scene recognition in familiar views. The results support the notion of dual effects that updating can occur automatically only as a result of bodily locomotion and it is hard to ignore this effect. Moreover, spatial updating, a long-term evolutionary ability, can be transferred to a new situation after a brief scene study. Lastly, VR technology can be a method with good validity in researching spatial updating of scene recognition.

We thank Dr Mou's great support for this research, Dr Roberts for proof reading and two reviewers' help for comments. This work was supported by the Autonomic Funding, Decision Making and Escape Behavior in Virtual Environment (2009THZ0), the Talented Faculty Start-up Funding of Tianjin Normal University (5RW092), and the Twelfth Five-Year Plan of Tianjin Science of Education Funding (CEYP505).

1 Sancaktar İ, Demirkan H. Spatial updating of objects after rotational and translational body movements in virtual environments. Comput Human Behav, 2008, 24: 2682-2696

2 Banta L P, Lecci S, Prętre V, et al. As the world turns: Short-term human spatial memory in egocentric and allocentric coordinates. Behav Brain Res, 2011, 219: 132-141

3 Wang R F, Brockmole J R. Simultaneous spatial updating in nested environments. Psychon Bull Rev, 2003, 10: 981-986

4 Wang R F, Spelke E S. Updating egocentric representations in human navigation. Cognition, 2000, 77: 215-250

5 Wang R F, Simons D J. Active and passive scene recognition across views. Cognition, 1999, 70: 191-210
6 Simons D J. In sight, out of mind: When object representations fail. Psychol Sci, 1996, 7: 301-305

7 Wang R F. Spatial representations and spatial updating. Psychol Learning Motiv, 2003, 42: 109-156

8 Farrell M J, Robertson I H. Mental rotation and automatic updating of body-centered spatial relationships. J Exp Psychol Learn Mem Cogn, 1998, 24: 227-233

9 Farrell M J, Thomson J A. Automatic spatial updating during locomotion without vision. Q J Exp Psychol A, 1998, 51: 637-654

10 Rieser J J. Access to knowledge of spatial structure at novel points of observation. J Exp Psychol Learn Mem Cogn, 1989, 15: 1157-1165

11 Meilinger T, Vosgerau G. Putting egocentric and allocentric into perspective. In: Höolscher C, Shipley T F, Belardinelli M O, et al., eds. Spatial Cognition VII: Lect Notes in Computer Science, Vol 6222. Berlin: Springer-Verlag, 2010. 207-221

12 Wan X I, Wang R F, Crowell J A. Spatial updating in superimposed real and virtual environments. Atten Percept Psychophys, 2009, 71: 42-51

13 Klatzky R L, Loomis J M, Beall A C, et al. Spatial updating of selfposition and orientation during real, imagined, and virtual locomotion. Psychol Sci, 1998, 9: 293-298

14 Riecke B E, Cunningham D W, Bülthoff H H. Spatial updating in virtual reality: The sufficiency of visual information. Psychol Res, 2007, 71: 298-313

15 May M, Klatzky R L. Path integration while ignoring irrelevant movement. J Exp Psychol Learn Mem Cogn, 2000, 26: 169-186

16 Wraga M, Creem-Regehr S H, Proffitt D R. Spatial updating of virtual displays. Mem Cognit, 2004, 32: 399-415

17 Simons D J, Wang R F. Perceiving real-world viewpoint changes. Psychol Sci, 1998, 9: 315-320

18 Wang R F, Simons D J. Active and passive scene recognition across views. Cognition, 1999, 70: 191-210

19 Wang R F, Brockmole J R. Simultaneous spatial updating in nested environments. Psychon Bull Rev, 2003, 10: 981-986

20 Mou W, Mcnamara T P. Intrinsic frames of reference in spatial memory. J Exp Psychol Learn Mem Cogn, 2002, 28: 162-170

21 Mou W, Li X, Mcnamara T P. Body-and environmental-stabilized processing of spatial knowledge. J Exp Psychol Learn Mem Cogn, 2008, 34: 415-421

22 Mou W, Mcnamara T P, Rump B, et al. Roles of egocentric and allocentric spatial representations in locomotion and reorientation. J Exp Psychol Learn Mem Cogn, 2006, 32: 1274-1290

23 Burgess N, Spiers H J, Paleologou E. Orientational manoeuvres in the dark: Dissociating allocentric and egocentric influences on spatial memory. Cognition, 2004, 94: 149-166

24 Blascovich J, Loomis J, Beall A C, et al. Target article: Immersive virtual environment technology as a methodological tool for social psychology. Psychol Inq, 2002, 13: 103-124

25 Fox J, Arena D, Bailenson J N. Virtual reality: A survival guide for the social scientist. J Med Psychol Theor Methods Applica, 2009, 21: 95-113

26 Loomis J M, Blascovich J J, Beall A C. Immersive virtual environment technology as a basic research tool in psychology. Behav Res Methods, 1999, 31: 557-564

27 Peng K P, Liu Y, Cao C M, et al. Virtual social psychology: Exploration and implication (in Chinese). Adv Psychol Sci, 2011, 19: 933-943

28 Lehmann A, Vidal M, Bülthoff H H. A high-end virtual reality setup for the study of mental rotations. Presence, 2008, 17: 365-375

29 Mou W, Mcnamara T P, Valiquette C M, et al. Allocentric and egocentric updating of spatial memories. J Exp Psychol Learn Mem Cogn, 2004, 30: 142-157

30 Zhao M, Zhou G, Mou W, et al. Spatial updating during locomotion does not eliminate viewpoint-dependent visual object processing. Vis Cogn, 2007, 15: 402-419

31 Mou W, Xiao C, Mcnamara T P. Reference directions and reference objects in spatial memory of a briefly viewed layout. Cognition, 2008, 108: $136-154$

32 Lloyd J, Persaud N V, Powell T E. Equivalence of real-world and 
virtual-reality route learning: A pilot study. Cyberpsychol Behav, 2009, 12: 423-427

$33 \mathrm{Hu} \mathrm{B}$, Ma L, Zhang W, et al. Predicting real-world ergonomic measurements by simulation in a virtual environment. Int $\mathrm{J}$ Ind Ergon, 2011, 41: 64-71

34 Parsons T D, Rizzo A A. Initial validation of a virtual environment for assessment of memory functioning: Virtual reality cognitive performance assessment test. Cyberpsychol Behav, 2008, 11: 17-25

35 Bayraktar F, Amca H. Interrelations between virtual-world and realworld activities: Comparison of genders, age groups, and pathological and nonpathological Internet users. Cyberpsychol Behav, 2012, 15: $263-269$

Open Access This article is distributed under the terms of the Creative Commons Attribution License which permits any use, distribution, and reproduction in any medium, provided the original author(s) and source are credited. 